01

\title{
Локализация и трансформация нелинейных возбуждений вблизи границы раздела сред с различными знаками нелинейности
}

\author{
(C) C.E. Савотченко
}

Белгородский государственный технологический университет им. В.Г. Шухова, 308012 Белгород, Россия

e-mail: savotchenkose@mail.ru

(Поступило в Редакцию 24 мая 2017 г.)

Рассмотрены контактные состояния на границе нелинейных сред с разными знаками ангармонизмов. Сформулирована модель, которая представляет собой контрактную краевую задачу для нелинейного уравнения Шредингера. В рассматриваемой системе в зависимости от значения энергии получены несколько типов стационарных состояний, описывающих локальные состояния вблизи границы раздела сред, локализацию нелинейных волн при переходе через границу, а также их трансформацию. Получены дисперсионные соотношения, определяющие значения энергии таких состояний. В предельных случаях получены выражения для энергии стационарных состояний в явном виде.

DOI: 10.21883/JTF.2019.02.47063.2355

\section{Введение}

Происходящие вблизи контакта двух сред с различными физическими свойствами процессы имеют большое значение в различных технических приложениях квантовой твердотельной электроники и оптоэлектроники. Существенную роль в этом играют исследования показателей прозрачности границ раздела сред, определяемые различными явлениями локализации возбуждений в кристаллах с плоскими дефектами. Для разработки средств управления в сложных электронных и оптических системах может представлять интерес создание контролируемых барьеров посредством границ раздела сред с различными физическими характеристиками, в частности - с различными параметрами ангармонизма межатомного взаимодействия.

Можно отметить большое количество теоретических работ, в которых при формулировке математических моделей активно используется нелинейное уравнение Шредингера (НУШ) [1,2], в том числе и для описания локализованных вблизи дефектов малоамплитудных нелинейных колебаний в ангармонических кристаллах [3]. С помощью НУШ в ангармонических кристаллах с дефектами также описаны локализованные колебательные состояния с частотами вне зоны сплошного спектра, зависящими от амплитуды колебаний [4].

Динамика полей различной физической природы, таких как упругого, электрического и магнитного, описывается НУШ. К примеру, НУШ применялось для описания локализации электромагнитных волн вблизи границ раздела нелинейных сред [5]. Кроме того, известны различные обобщения НУШ, в частности, для среды с пространственной дисперсией использовано НУШ с производными четвертого порядка [6] и короткодействующим потенциалом с дельта-функцией Дирака, моделирующей взаимодействие возбуждения с дефектом. В [7] было получено решение НУШ с потенциалом, моделирующим взаимодействие возбуждения с дефектом, обладающим внутренней структурой, при учете дальнодействующих сил межатомного взаимодействия.

Для теоретического исследования новых явлений, обусловленных локализацией возбуждений различной физической природы вблизи дефектов, целесообразно использовать НУШ, которому подчиняется волновая функция $\psi$, выступающая в роли огибающей комплексного поля компонент вектора намагниченности в легкоосном ферромагнетике, либо комплексной амплитуды упругого поля смещения сдвиговой волны в кубическом кристалле с плоским дефектом, либо комплексной функции из амплитуд компонент электрического поля в оптической нелинейной среде. Параметры в уравнениях, описывающих указанные виды полей, будут иметь вполне определенный физический смысл в рамках одной из трех таких моделей.

В настоящей работе предлагается описать различные виды стационарных состояний, которые возникают вблизи границы нелинейных сред, параметр ангармонизма в которых имеет противоположные знаки. Такие состояния являются различными видами решениями НУШ с короткодействующим потенциалом, связанные на границе раздела сред. Волновые функции в таких состояниях в зависимости от энергии возбуждений в полупространстве одной среды могут быть затухающими при удалении от границы раздела, а в другой среде с противоположным знаком ангармонизма они могут иметь форму кноидальных волн, соответствующих периодическим решениям НУШ. Комбинации различных типов решений НУШ, связанных граничными условиями на дефекте, приводят к многообразию различных типов стационарных состояний, описывающих явления локализации и трансформации нелинейных волн при переходе через границу раздела сред. 


\section{Формулировка математической модели}

Рассмотрим контакт двух нелинейных сред с различными физическими характеристиками, ангармонизм в которых имеет противоположные знаки. Среды разделены плоской границей раздела, проходящей через начало координат, перпендикулярно оси $O x$. Полупространство в области $x<0$ занимает среда с одним знаком параметра ангармонического межатомного взаимодействия $\gamma$, а полупространство в области $x>0$ занимает среда с противоположным знаком этого параметра.

Будем предполагать, что возмущение параметров сред создаваемой границей раздела как плоским дефектом сосредоточено на расстояниях, существенно меньших размеров изучаемых возбуждений, и поэтому считается локальным. Вследствие локальности возмущения параметров сред взаимодействие границы раздела с возбуждением можно описывать короткодействующим потенциалом $U(x)=U_{0} \delta(x)$, где $\delta(x)-\delta$-функция Дирака, $U_{0}$ - интенсивность взаимодействия дефекта, расположенным в начале координат, с возбуждением, такая, что при $U_{0}>0$ возбуждение отталкивается от дефекта, а при $U_{0}<0$ - притягивается.

Будем рассматривать процессы локализации и трансформации возбуждений на границе раздела линейной и нелинейной сред на основе НУШ.

Стационарные состояния НУШ с энергией $E$ представимы виде: $\psi(x, t)=\psi(x) \exp (-i E t)$. Тогда задача решения НУШ с указанным короткодействующим потенциалом сводится к решению стационарного одномерного НУШ на полуосях:

$E \psi(x)=-\frac{1}{2 m} \psi^{\prime \prime}(x)+\Omega(x) \psi(x)-\gamma(x)|\psi(x)|^{2} \psi(x)=0$,

с граничными условиями:

$$
\begin{gathered}
\psi(-0)=\psi(+0) ; \\
\psi^{\prime}(+0)-\psi^{\prime}(-0)=2 m U_{0} \psi(0) .
\end{gathered}
$$

Здесь $m$ - эффективная масса возбуждений,

$$
\Omega(x)=\left\{\begin{array}{ll}
\Omega_{1}, & x<0 \\
\Omega_{2}, & x>0
\end{array},\right.
$$

$\Omega_{1,2}$ - значения уровней дна энергетический зоны, $\gamma(x)$ - параметр нелинейности сред:

$$
\gamma(x)=\left\{\begin{array}{ll}
\gamma_{1}, & x<0 \\
-\gamma_{2}, & x>0
\end{array},\right.
$$

причем $\gamma_{1,2}>0$.

В линейной среде без дефекта распространяются свободные волны с квадратичным законом дисперсии. В нелинейной среде без дефекта могут распространяться локализованные возбуждения - солитоны, и нелинейные волны, описываемые периодическими решениями
НУШ, которые выражаются через эллиптические функции и называются кноидальными волнами $[1,2]$. В зависимости от знака нелинейности существуют различные типы нелинейных волн.

\section{Локализованные состояния}

В рассматриваемой модели существует несколько типов локализованных состояний в различных энергетических диапазонах. В области ниже дна зоны любой из ветвей спектра, когда энергия возбуждений находится в диапазоне $E<\min \left(\Omega_{1,2}\right)$, уравнение (1) имеет решение, удовлетворяющее граничным условиям (2) и (3), представимое в виде:

$$
\psi(x)=\left\{\begin{array}{ll}
A_{1} / \operatorname{ch} q_{1}\left(x-x_{1}\right), & x<0 \\
A_{2} / \operatorname{sh} q_{2}\left(x-x_{2}\right), & x>0
\end{array},\right.
$$

где $\quad q_{1,2}^{2}=2 m\left(\Omega_{1,2}-E\right), \quad$ амплитуды $\quad A_{1,2}= \pm q_{1,2} /$ $\left(m \gamma_{1,2}\right)^{1 / 2}$. Для ограниченности решения (4) должно выполняться требование $x_{2}<0$. Из граничных условий (2) и (3) получаются два соотношения, позволяющие определить энергию данного стационарного состояния, и один из параметров $x_{1,2}$. К примеру, можно выразить

$$
x_{2}=\frac{1}{q_{2}} \operatorname{Arsh}\left(\frac{q_{2}}{\eta q_{1}} \operatorname{ch} q_{1} x_{1}\right),
$$

где $\eta=\left(\gamma_{2} / \gamma_{1}\right)^{1 / 2}$. Тогда значения энергии определяются из дисперсионного соотношения, полученного с учетом (5):

$$
q_{2} \operatorname{cth}\left(q_{2} x_{2}\right)-q_{1} \operatorname{th}\left(q_{1} x_{1}\right)=2 m U_{0} .
$$

Из соотношения (6) с учетом (5) находится энергия как функция параметров $E=E\left(m, U_{0}, \eta, \Omega_{1,2}, x_{1}\right)$, а соотношение (5) тогда определяет параметр $x_{2}$. Таким образом, решение (4) является однопараметрическим локализованным состоянием с одним свободным параметром, в качестве которого удобно было выбрать $x_{1}$.

В явном виде энергию из (6) можно получить для частного случая $x_{1}=0$ :

$$
E=\Omega_{1}-\left(2 m U_{0}\right)^{2} /\left(1+\eta^{2}\right) .
$$

В диапазоне $\Omega_{2}<E<\Omega_{1}$, что возможно при условии $\Omega_{2}<\Omega_{1}$, уравнение (1) имеет решение, удовлетворяющее граничным условиям (2) и (3), представимое в виде:

$$
\psi(x)=\left\{\begin{array}{ll}
A_{1} / \operatorname{ch} q_{1}\left(x-x_{1}\right), & x<0 \\
A_{t} \operatorname{th} q_{t}\left(x-x_{2}\right), & x>0
\end{array},\right.
$$

где параметры волновой функции в области $x<0$ такие же, как для (4), а в области $x>0 \quad q_{t}^{2}=m\left(E-\Omega_{2}\right)$, $A_{t}= \pm q_{t} /\left(m \gamma_{2}\right)^{1 / 2}$. Подстановка (8) в граничные условия (2) и (3) приводит к соотношениям

$$
\begin{gathered}
\operatorname{ch} q_{1} x_{1} \text { th } q_{t} x_{2}=\eta q_{1} / q_{t}, \\
q_{t} / \operatorname{sh} 2 q_{t} x_{2}+q_{1} \text { th } q_{1} x_{1}=-2 m U_{0} .
\end{gathered}
$$


Из соотношения (9) выражается параметр $x_{2}$, а из (10) находится энергия как функция параметров $E=E\left(m, U_{0}, \eta, \Omega_{1,2}, x_{1}\right)$. Поэтому волновая функция (8) описывает однопараметрическое локализованное состояние с одним свободным параметром, в качестве которого выбран $x_{1}$.

В частном случае при $x_{1}=0$ из (10) при учете (9) получается соотношение

$$
q_{t}^{2}+q_{1}^{2} \eta^{2}=2 m U_{0} \eta q_{1},
$$

из которого можно получить энергию в явном виде

$$
E=\left(b \pm D^{1 / 2}\right) /\left(1-\eta^{2}\right)^{2}
$$

где $\quad b=\Omega_{2}-2\left(\Omega_{1}+2 m U_{0}^{2}\right) \eta^{2}, \quad D=b^{2}-\left(1-\eta^{2}\right)^{2} \times$ $\times\left\{\left(\Omega_{2}-2 \Omega_{1} \eta^{2}\right)^{2}-8 m U_{0}^{2} \eta^{2} \Omega_{1}\right\}$.

Знак „, ${ }^{“}$ в (12) выбирается для одних соотношений между параметрами системы, а „_-“ - для других.

\section{Локализация нелинейных волн}

В рассматриваемой системе могут существовать состояния, описывающие локализацию нелинейных волн при переходе через границу раздела сред.

В диапазоне $\Omega_{2}<E<\Omega_{1}$ уравнение (1) имеет решение, удовлетворяющее граничным условиям (2) и (3), представимое в виде:

$$
\psi(x)=\left\{\begin{array}{ll}
A_{1} / \operatorname{ch} q_{1}\left(x-x_{1}\right), & x<0 \\
A_{s} \operatorname{sn}\left(q_{s}\left(x-x_{2}\right), k\right), & x>0
\end{array},\right.
$$

где параметры волновой функции в области $x<0$ такие же, как для (4), а в области $x>0 A_{s}= \pm q_{s}\left(m \gamma_{2}\right)^{-1 / 2}$, $q_{s}^{2}=2 m\left(E-\Omega_{2}\right) /\left(1+k^{2}\right), k-$ модуль эллиптической функции sn $(0<k<1)$. Подстановка (13) в граничные условия (2) и (3) приводит к соотношениям

$$
\operatorname{ch} q_{1} x_{1} \operatorname{sn}\left(q_{s} x_{x}, k\right)=\eta q_{1} / q_{s},
$$

$q_{s} k_{1} \operatorname{cn}\left(q_{s} x_{2}, k\right) / \operatorname{cn}\left(q_{s} x_{2}+K(k), k\right)-q_{1}$ th $q_{1} x_{1}=2 m U_{0}$,

$k_{1}^{2}=1-k^{2}-$ дополнительный модуль эллиптических функций, $K(k)$ - полный эллиптический интеграл первого рода [1].

Из (14) можно выразить $x_{1}$ и подставить в (15), в результате чего получится соотношение

$$
\begin{aligned}
& q_{1}\left(q_{s}^{2} \operatorname{sn}\left(q_{s} x_{2}, k\right)+\eta^{2} q_{1}^{2}\right)^{1 / 2}=-q_{s}\left\{2 m U_{0} \operatorname{sn}\left(q_{s} x_{2}, k\right)\right. \\
& \left.\quad+q_{s} \operatorname{cn}\left(q_{s} x_{2}, k\right) \operatorname{dn}\left(q_{s} x_{2}, k\right)\right\} .
\end{aligned}
$$

Из соотношения (16) находится энергия как функция параметров $E=E\left(m, U_{0}, \eta, \Omega_{1,2}, x_{2}, k\right)$, а соотношение (15) тогда определяет параметр $x_{1}$. Таким образом, волновая функция (13) является двухпараметрическим решением с двумя свободными параметрами, в качестве которых выбраны $k$ и $x_{2}$.
Рассмотрим предельный случай малых значений $q_{s} x_{2} \ll 1$ и $x_{1}=0$. Тогда получается $x_{2}=-1 / 2 m U_{0}$. С учетом данного выражения в таком пределе можно определить энергию в явном виде

$$
E=\alpha_{s}\left\{1+\left[1+\beta_{s} / \alpha_{s}^{2}\right]^{1 / 2}\right\}^{2},
$$

где $\alpha_{s}=\Omega_{2}-m U_{0}^{2} \eta^{2}\left(1+k^{2}\right)^{2}, \beta_{s}=\Omega_{2}^{2}-m U_{0}^{2} \eta^{2}\left(1+k^{2}\right)^{2} \Omega_{1}$.

Состояние (13) описывает эффект „запирания“ волны при переходе из правого полупространства $(x>0)$ в левое $(x<0)$. Кноидальная волна, распространяющаяся в полупространстве со средой, характеризуемой отрицательным ангармонизмом, при переходе через границу раздела в правом полупространстве со средой с отрицательным ангармонизмом, затухает, причем на больших расстояниях от нее $|\psi| \rightarrow 0$ при $x \rightarrow-\infty$.

В рассматриваемом энергетическом диапазоне могут существовать два типа состояний, волновые функции которых описываются периодическими решениями НУШ в левом полупространстве со средой с положительным ангармонизмом, и решением в виде кинка в правом полупространстве со средой с отрицательным ангармонизмом. Волновая функция одного из таких состояний является решением уравнения (1), удовлетворяющим граничным условиям (2) и (3), представимым в виде

$$
\psi(x)=\left\{\begin{array}{ll}
A_{d} \operatorname{dn}\left(q_{d}\left(x-x_{1}\right), k\right) & x<0 \\
A_{t} \operatorname{th} q_{t}\left(x-x_{2}\right) & x>0
\end{array},\right.
$$

где параметры волновой функции в области $x>0$ такие же, как для (8), а $A_{q}= \pm q_{d} /\left(m \gamma_{1}\right)^{1 / 2}$, $q_{d}^{2}=2 m\left(\Omega_{1}-E\right) /\left(2-k^{2}\right)$. Подстановка (18) в граничные условия (2) и (3) приводит к соотношениям

$$
\begin{gathered}
\eta \operatorname{cth} q_{t} x_{2} \operatorname{dn}\left(q_{d} x_{1}, k\right)=q_{t} / q_{d}, \\
q_{t} / \operatorname{ch}^{2} q_{t} x_{2}+\operatorname{th} q_{t} x_{2}\left\{k^{2} q_{d} \operatorname{sn}\left(q_{d} x_{1}, k\right)\right. \\
\left.\times \operatorname{sn}\left(q_{d} x_{1}+K(k), k\right)+2 m U_{0}\right\}=0 .
\end{gathered}
$$

Из (19) можно выразить $x_{2}$ и подставить в (20), в результате чего получится соотношение

$$
\begin{aligned}
\eta q_{d}^{2} & \left\{\eta \operatorname{dn}^{2}\left(q_{d} x_{1}, k\right)+k^{2} \operatorname{sn}\left(q_{d} x_{1}, k\right) \operatorname{cn}\left(q_{d} x_{1}, k\right)\right\}+q_{t}^{2} \\
& =2 m U_{0} \eta q_{d} \operatorname{dn}\left(q_{d} x_{1}, k\right)
\end{aligned}
$$

Из соотношения (21) находится энергия как функция параметров $E=E\left(m, U_{0}, \eta, \Omega_{1,2}, x_{1}, k\right)$, а соотношение (19) тогда определяет параметр $x_{2}$. Волновая функция (18) является двухпараметрическим решением с двумя свободными параметрами, в качестве которых выбраны $k$ и $x_{1}$.

В частном случае при $x_{1}=0$ из (21) при учете (19) получается соотношение

$$
q_{t}^{2}+q_{d}^{2} \eta^{2}=2 m U_{0} q_{d}
$$


из которого можно получить энергию в явном виде:

$$
E=\Omega_{1}-\varepsilon_{d}\left\{1 \pm\left[1-\Omega_{d} / \varepsilon_{d}\right]^{1 / 2}\right\}^{2} .
$$

где $\quad \varepsilon_{d}=\eta U_{0}\left(2-k^{2}\right) /\left[2 \eta^{2}-\left(2-k^{2}\right)\right], \quad \Omega_{d}=\left(\Omega_{1}-\right.$ $\left.-\Omega_{2}\right)\left(2-k^{2}\right) / 2 m U_{0} \eta$. Знаки $\Omega_{d}$ и $\varepsilon_{d}$ определяются только знаком параметра дефекта $U_{0}$ и поэтому $\Omega_{d} / \varepsilon_{d}>0$. Должно выполняться требование $\Omega_{d}<\varepsilon_{d}$.

В рассматриваемом энергетическом диапазоне уравнение (1) имеет решение, удовлетворяющим граничным условиям (2) и (3), представимое в виде

$$
\psi(x)=\left\{\begin{array}{ll}
A_{c} \operatorname{cn}\left(q_{c}\left(x-x_{1}\right), k\right) & x<0 \\
A_{t} \operatorname{th}\left(q_{t}\left(x-x_{2}\right)\right), & x>0
\end{array},\right.
$$

где параметры волновой функции в области $x>0$ такие же, как для (8), а $A_{c}= \pm k q_{c} /\left(m \gamma_{1}\right)^{1 / 2}$, $q_{c}^{2}=2 m\left(\Omega_{1}-E\right) /\left(2 k^{2}-1\right)$. Подстановка (24) в граничные условия (2) и (3) приводит к соотношениям

$$
\begin{gathered}
\eta \operatorname{cth} q_{t} x_{2} \operatorname{cn}\left(q_{c} x_{1}, k\right)=q_{t} / q_{c}, \\
q_{t} / \operatorname{ch}^{2} q_{t} x_{2}+\operatorname{th} q_{t} x_{2}\left\{q_{c} \operatorname{sn}\left(q_{c} x_{1}, k\right) / \operatorname{sn}\left(q_{c} x_{1}+K(k), k\right)\right. \\
\left.+2 m U_{0}\right\}=0 .
\end{gathered}
$$

Из (25) можно выразить $x_{2}$ и подставить в (26), в результате чего получится соотношение

$$
\begin{aligned}
& \eta q_{c}^{2}\left\{\eta \operatorname{cn}^{2}\left(q_{c} x_{1}, k\right)-k \operatorname{sn}\left(q_{c} x_{1}, k\right) \operatorname{dn}\left(q_{c} x_{1}, k\right)\right\}+q_{t}^{2} \\
& =2 m U_{0} \eta q_{c} k \operatorname{cn}\left(q_{c} x_{1}, k\right) .
\end{aligned}
$$

Из соотношения (27) находится энергия как функция параметров $E=E\left(m, U_{0}, \eta, \Omega_{1,2}, x_{1}, k\right)$, а соотношение (25) тогда определяет параметр $x_{2}$. Волновая функция (24) является двухпараметрическим решением с двумя свободными параметрами, в качестве которых выбраны $k$ и $x_{1}$.

В частном случае при $x_{1}=0$ из (27) при учете (25) получается соотношение

$$
q_{c}^{2} \eta^{2}+q_{t}^{2}=2 m U_{0} \eta q_{c},
$$

из которого можно получить энергию в явном виде

$$
E=\Omega_{1}-\varepsilon_{c}\left\{1 \pm\left[1-\Omega_{c} / \varepsilon_{c}\right]^{1 / 2}\right\}^{2} .
$$

где $\quad \varepsilon_{c}=2 m \eta^{2} U_{0}^{2}\left(2 k^{2}-1\right) /\left[\left(2 \eta^{2}-\left(2 k^{2}-1\right)\right]^{2}, \quad \Omega_{c}=\right.$ $=\left(\Omega_{1}-\Omega_{2}\right)\left(2 k^{2}-1\right) /\left[2 \eta^{2}\left(2 k^{2}-1\right)\right]$. Должно выполняться требование $\Omega_{c}<\varepsilon_{c}$.

Ниже границы сплошного спектра в диапазоне $E<\min \left(\Omega_{1,2}\right)$ также существуют еще два вида состояний, описывающих локализацию кноидальных волн при переходе через границу раздела.

Уравнение (1) в данном энергетическом диапазоне имеет решение, удовлетворяющее граничным условиям (2) и (3), представимое в виде

$$
\psi(x)= \begin{cases}A_{d} \operatorname{dn}\left(q_{d}\left(x-x_{1}\right), k\right) & x<0 \\ A_{2} / \operatorname{sh} q_{2}\left(x-x_{2}\right), & x>0\end{cases}
$$

где параметры волновой функции в области $x<0$ такие же, как для (18), а в области $x>0$ такие же, как для (4). Подстановка (30) в граничные условия (2) и (3) приводит к соотношениям

$$
\eta \operatorname{sh} q_{2} x_{2} \operatorname{dn}\left(q_{d} x_{1}, k\right)=q_{2} / q_{d} .
$$

$q_{2}$ th $q_{2} x_{2}+k^{2} q_{d} \operatorname{sn}\left(q_{d} x_{1}, k\right) \operatorname{sn}\left(q_{d} x_{1}+K(k), k\right)+2 m U_{0}=0$.

Из (31) можно выразить $x_{2}$ и подставить в (32), тем самым исключив его из дисперсионного соотношения. Тогда из (32) находится энергия как функция параметров $E=E\left(m, U_{0}, \eta, \Omega_{1,2}, x_{1}, k\right)$, а (31) определяет параметр $x_{2}$. Поэтому волновая функция (30) является двухпараметрическим решением НУШ с двумя свободными параметрами, в качестве которых выбраны $k$ и $x_{1}$.

В частном случае при $x_{1}=0$ из (32) при учете (31) можно получить энергию в явном виде

$$
E=\Omega_{1}-\mu_{d}\left(\Omega_{2}-\Omega_{1}\right),
$$

где $\mu_{d}=\left(4 m^{2} U_{0}^{2}-1\right)\left(2-k^{2}\right) /\left[2-k^{2}-4 m^{2} U_{0}^{2}\left(2-k^{2}-\right.\right.$ $\left.-\eta^{2}\right)$ ]. Если $\Omega_{1}>\Omega_{2}$, то должно быть $\mu_{d}<0$, а если $\Omega_{1}<\Omega_{2}$, то должно быть $\mu_{d}>0$.

Уравнение (1) в рассматриваемом энергетическом диапазоне $E<\min \left(\Omega_{1,2}\right)$ имеет решение, удовлетворяющее граничным условиям (2) и (3), представимое в виде

$$
\psi(x)=\left\{\begin{array}{ll}
A_{c} \operatorname{cn}\left(q_{c}\left(x-x_{1}\right), k\right) & x<0 \\
A_{2} / \operatorname{sh}\left(q_{2}\left(x-x_{2}\right)\right), & x>0
\end{array},\right.
$$

где параметры волновой функции в области $x<0$ такие же, как для (24), а в области $x>0$ такие же, как для (4). Подстановка (34) в граничные условия (2) и (3) приводит к соотношениям

$$
k \eta \operatorname{sh} q_{2} x_{2} \operatorname{cn}\left(q_{c} x_{1}, k\right)=q_{2} / q_{c},
$$

$q_{2} \operatorname{cth} q_{2} x_{2}-q_{c} \operatorname{sn}\left(q_{c} x_{1}, k\right) / \operatorname{sn}\left(q_{c} x_{1}+K(k), k\right)-2 m U_{0}=0$.

Из (35) можно выразить $x_{2}$ и подставить в (36), тем самым исключив его из дисперсионного соотношения. Тогда из (36) находится энергия как функция параметров $E=E\left(m, U_{0}, \eta, \Omega_{1,2}, x_{1}, k\right)$, а (35) определяет параметр $x_{2}$. Поэтому волновая функция (34) является двухпараметрическим решением НУШ с двумя свободными параметрами, в качестве которых выбраны $k$ и $x_{1}$.

В частном случае при $x_{1}=0$ из (36) при учете (35) можно получить энергию в явном виде

$$
E=\Omega_{1}-\left(\Omega_{1}-\Omega_{2}+2 m U_{0}^{2}\right)\left(2 k^{2}-1\right) /\left[k^{2}\left(2+\eta^{2}\right)-1\right] .
$$

\section{Трансформация нелинейных волн}

В рассматриваемой системе в диапазоне $\Omega_{2}<E<\Omega_{1}$ могут существовать состояния, описывающие трансформацию нелинейных волн при переходе через границу раздела сред. 
Уравнение (1) в данном энергетическом диапазоне имеет решение, удовлетворяющее граничным условиям (2) и (3), представимое в виде

$$
\psi(x)=\left\{\begin{array}{ll}
A_{d} \operatorname{dn}\left(q_{d}\left(x-x_{1}\right), k\right) & x<0 \\
A_{s} \operatorname{sn}\left(q_{s}\left(x-x_{2}\right), k\right), & x>0
\end{array},\right.
$$

где параметры волновой функции в области $x<0$ такие же, как для (18), а в области $x>0$ такие же, как для (13). Подстановка (30) в граничные условия (2) и (3) приводит к соотношениям

$$
\begin{gathered}
\operatorname{sn}\left(q_{s} x_{2}, k\right) / \operatorname{dn}\left(q_{d} x_{1}, k\right)=\eta q_{d} / q_{s}, \\
q_{s} k_{1} \operatorname{cn}\left(q_{s} x_{2}, k\right) / \operatorname{cn}\left(q_{s} x_{2}+K(k), k\right) \\
-k^{2} q_{d} \operatorname{sn}\left(q_{d} x_{1}, k\right) \operatorname{sn}\left(q_{d} x_{1}+K(k), k\right)=2 m U_{0} .
\end{gathered}
$$

Из (39) можно выразить, например $x_{2}$, и подставить в (40), тем самым исключив его из дисперсионного соотношения. Тогда из (40) находится энергия как функция параметров $E=E\left(m, U_{0}, \eta, \Omega_{1,2}, x_{1}, k\right)$, а (39) определяет параметр $x_{2}$. Поэтому волновая функция (38) является двухпараметрическим решением НУШ с двумя свободными параметрами, в качестве которых выбраны $k$ и $x_{1}$.

В предельном случае малых значений $q_{s} x_{2} \ll 1$ и $x_{1}=0$ из (40) при учете (39) можно получить энергию в явном виде

$$
E=\Omega_{2}+\varepsilon_{d s}\left\{\left[1+2\left(\Omega_{1}-\Omega_{2}\right) / \varepsilon_{d s}\right]^{1 / 2}-1\right\},
$$

где $\varepsilon_{d s}=m U_{0}^{2} \eta^{2}\left(1+k^{2}\right)^{2} /\left(2-k^{2}\right)>0$.

Уравнение (1) в данном энергетическом диапазоне имеет решение, удовлетворяющее граничным условиям (2) и (3), другого типа, которое представимо в виде

$$
\psi(x)=\left\{\begin{array}{ll}
A_{c} \operatorname{cn}\left(q_{c}\left(x-x_{1}\right), k\right) & x<0 \\
A_{s} \operatorname{sn}\left(q_{s}\left(x-x_{2}\right), k\right), & x>0
\end{array},\right.
$$

где параметры волновой функции в области $x<0$ такие же, как для (24), а в области $x>0$ такие же, как для (13). Подстановка (42) в граничные условия (2) и (3) приводит к соотношениям

$$
\begin{gathered}
k \operatorname{cn}\left(q_{c} x_{1}, k\right) / \operatorname{sn}\left(q_{s} x_{2}, k\right)=\eta q_{s} / q_{c}, \\
q_{s} k_{1} \operatorname{cn}\left(q_{s} x_{2}, k\right) / \operatorname{cn}\left(q_{s} x_{2}+K(k), k\right) \\
-q_{c} \operatorname{sn}\left(q_{c} x_{1}, k\right) / \operatorname{sn}\left(q_{c} x_{1}+K(k), k\right)=2 m U_{0} .
\end{gathered}
$$

Из (43) можно выразить, например, $x_{2}$ и подставить в (44), тем самым исключив его из дисперсионного соотношения. Тогда из (44) находится энергия как функция параметров $E=E\left(m, U_{0}, \eta, \Omega_{1,2}, x_{1}, k\right)$, а (43) определяет параметр $x_{2}$. Поэтому волновая функция (42) является двухпараметрическим решением НУШ с двумя свободными параметрами, в качестве которых выбраны $k$ и $x_{1}$.
В предельном случае малых значений $q_{s} x_{2} \ll 1$ и $x_{1}=0$ из (40) при учете (39) можно получить энергию в явном виде

$$
E=\Omega_{2}+\varepsilon_{c s}\left\{\left[1+2\left(\Omega_{1}-\Omega_{2}\right) / \varepsilon_{c s}\right]^{1 / 2}-1\right\},
$$

где $\varepsilon_{c s}=m U_{0}^{2} \eta^{2} k^{2}\left(1+k^{2}\right)^{2} / k_{1}^{2}\left(2 k^{2}-1\right)>0$.

Волновые функции (38) и (42) описывают трансформацию кноидальных волн при переходе через границу раздела нелинейных сред с различными знаками ангармонизма в диапазоне энергий $\Omega_{2}<E<\Omega_{1}$.

\section{Заключение}

В работе на основе модели контакта двух нелинейных сред, возбуждения в которой описываются НУШ, рассмотрены вопросы существования различных видов стационарных состояний в широком интервале энергетического спектра.

Получены новые решения сформулированной контактной краевой задачи для НУШ, описывающие взаимодействие возбуждений на границе раздела нелинейных сред с противоположными знаками параметра ангармонизма. Показано, что в зависимости от соотношения энергии возбуждения и значений краев зоны сплошного спектра собственных стационарных состояний возникают локализованные по обе стороны от границы раздела сред состояния. Установлено, что в рассматриваемой системе, помимо солитонных, возможны периодические решения НУШ, описывающие трансформацию кноидальных волн при переходе через границу раздела нелинейных сред с противоположными знаками параметра ангармонизма.

Особый интерес представляют такие решения контактной краевой задачи для НУШ, которые описывают локализацию кноидальных волн при переходе через границу раздела. В этом случае волновая функция в одном полупространстве представляет собой кноидальную волну, описываемую периодическим решением НУШ в виде определенной эллиптической функции, а другом полупространстве - затухающую при удалении от границы раздела функцию, описываемую солитонным решением НУШ. В частных предельных случаях получены выражения для энергии стационарных состояний в явном виде.

\section{Список литературы}

[1] Давыдов А.С. Солитоны в молекулярных системах. Киев: Наукова думка, 1984. 288 с.

[2] Косевич А.М., Ковалев А.С. Введение в нелинейную физическую механику. Киев: Наукова думка, 1989. 304 с.

[3] Герасимчук И.В., Ковалев А.С. // ФНТ. 2000. Т. 26. № 8. C. 799-809.

[4] Богдан М.М., Герасимчук И.В., Ковалев А.С. // ФНТ. 1997. T. 23. № 2. C. 197-207. 
[5] Abdullaev F.Kh., Baizakov B.B., Umarov B.A. // Opt. Commun. 1998. Vol. 156. P. 341-346.

[6] Савотченко С.E. // Известия вуз. Физика. 2004. Т. 47. № 5. C. $79-84$.

[7] Савотченко C.E. // Вестник Воронежского гос. ун-та. Серия: Физика. Математика. 2016. № 4. С. 51-59. 